slight green color. The tests were not as decided as those on the No. I lard but still distinctly visible. These results are probably due to the presence of the large percentage of No. I lard which the Western lard contains.

I night say further that lard rendered from the leaf alone, which does not, as far as I know, find its way to the lard market in this country, does not give a green color with the reagent. This fact may have been the cause of the many favorable endorsements which appeared after the first publication of the reaction. In order for the chemist to make a fair trial of the reagent, what, apparently, would appear fairer than to obtain a leaf and render it in the laboratory and make tests upon this lard?

In conclusion, I would say, that for the detection of less than ten to fifteen per cent. of cottonseed-oil the test is unreliable, as, in such an instance, no reaction may be produced. When a slight green is the result of a test, the presence of cottonseed-oil is not proven. When a heavy green color, deep emerald in shade, is obtained, the addition of a vegetable oil in considerable amounts is fairly certain. But it is easy to detect such large additions by other methods, and the phosphomolybdic test may be considered to be of slight analytical value and certainly does not fill that long-felt want of a simple test which will reliably indicate small percentages of cottonseed or other vegetable oils in lards.

I,ABORATORY OF TIH: CENTRAL LARD COMPANY,

Octobet 29,1894 .

\title{
SOME NEW SOLVENTS FOR PERCHROMIC ACID.'
}

\author{
By William M. Grosvenor, Jr. \\ Received Novenber 8,1894 .
}

( )

$\mathrm{N}$ the addition of hydrogen peroxide to an aqueous solution of potassium dichromate acidified with sulphuric acid, a blue color is produced, which is supposed to be caused by the formation of perchromic acid. Ether dissolves the blue substance, forming an intensely colored, deep blue solution. On standing, the perchromic acid in this solution decomposes with the formation of chromic acid or salts of chromium.

Recently, Griggi ( $J$. Chem. Soc., 64, 2,223) has stated that

I Read before the New York Sectiou, December $8,1893$. 
perchromic acid is also soluble in anyl alcohol, and that this solution is more stable than the ethereal solution, the color lasting for six hours. With a view to studying the behavior of perchromic acid toward certain substances, various organic liquids were tried as solvents for perchromic acid. A two per cent. solution of potassium dichromate was slightly acidified with sulphuric acid and then shaken with the solvent and hydrogen peroxide, after which it was allowed to stand. The following substances failed to dissolve the perchromic acid:

Carbon disulphide, benzene, ligroin, spirits of turpentine, castor oil, oil of wintergreen, oil of bergamot, kerosene, paraffin oils, chloroform, carbon tetrachloride, toluene, nitrobenzene, and aniline.

The following substances were found to be solvents for perchromic acid:

Ethyl acetic ester, valerianic ether, amyl valerianic ester, amyl chloride, amyl butyric ester, amyl formic ester, and anyl acetic ester.

In order to determine which of the solutions of perchromic acid lasted the longest, the following experinents were made. Equal announts of a dilute solution of chromic acid were shaken in the presence of the solvents with equal amounts of hydrogen peroxide, in small oil-sample bottles, and allowed to stand on a window frame exposed to a strong light. The temperature varied from $10^{\circ}-20^{\circ} \mathrm{C}$. The results were as follows:

Anyl alcohol retained its color one hour and ten minutes, then turned brown.

Anyl chloride retained its color one hour and fifty-five minutes, then turned colorless.

Amyl butyric ester retained its color one hour and fifty-five minutes, then turned brown.

Anyl valerianic ester retained its color two hours and ten minutes, then turned green.

Valerianic ether retained its color two hours and thirty-five miuutes, then turned colorless.

Amyl formic ester retained its color sixteen hours and fifty-five minutes, then turned white.

Anyl acetic ester retained its color seventeen hours and fifty-five minutes, then turnerl colorless.

Ethyl ether retained its color eighteen hours and twenty-five minutes, then turned colorless. 
Ethyl acetic ester retained its color twenty-three hours, then turned colorless.

These results do not agree with the observations of Griggi, that the amyl alcohol solution of perchromic acid is more stable than the solution in ether. The most stable solution is that in ethyl acetic ester. The time of duration given by Griggi, six hours, was evidently found under different circumstances. If the layer of the solvent is allowed to remain upon the aqueous layer the decomposition of the perchromic acid is greatly accelerated. The above experiments were made in this manner. Those of Griggi may, perhaps, have been made under other conditions in this respect.

CHEMICAL LABORATORY OF IHE BROORLYN POLYTECHNIC INSTITUTE.

\section{SOME FACTS OBSERVED IN THE DETERMINATION OF PHOSPHORIC ACID BY THE MOLYBDIC ACID PROCESS.}

\section{BY RUDOLF DE ROODE.} Received November 26,1894 .

THE length of time necessary for the complete precipitation supposed. Portions of the same solution were treated with molybdic solution and allowed to stand at $65^{\circ} \mathrm{C}$. for five, ten. fifteen, twenty, thirty, forty, fifty, and sixty minutes, respectively, and two portions stood in a warm place over night. The phosphoric acid determined as usual as magnesium pyrophosphate, showed that the precipitation was complete after standing only five minutes. The weights of magnesium pyrophosphate were as follows:

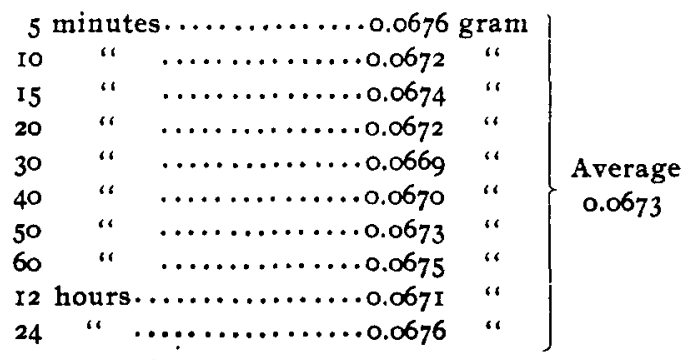

By making a determination of phosphoric acid, using twenty- 